\title{
Familiarity as a Factor in Designing Finger Gestures for Elderly Users
}

\author{
Christian Stößel \\ Center of Human-Machine Systems \\ Berlin Institute of Technology \\ Franklinstr. 28/29, FR 2-6 \\ 10587 Berlin \\ chs@zmms.tu-berlin.de
}

\begin{abstract}
Older adults often struggle with everyday technology which is not designed to accommodate their special needs and adapted to their specific cognitive, sensory and motor abilities. Recently, a new generation of mobile devices has been equipped with multitouch and acceleration sensors that allow for novel ways of interacting with these devices. Interaction occurs through gestures, such as finger movements on the device surface or movement patterns of the device itself, which trigger the device functions. It remains unclear, however, whether this gesture-based interaction indeed facilitates technology interaction, especially with regard to elderly users, or whether it further decreases accessibility and usability of such devices for this user group. In the present study we compare younger and older users on a set of 42 simple gestures with varying complexity and available gesture space regarding accuracy and velocity scales. Results indicate that older users are slower, but not necessarily less accurate, and that further factors such as familiarity can influence gesture performance differentially for older and younger users.
\end{abstract}

\section{Categories and Subject Descriptors}

H.5.2 [Information Interfaces and Presentation]: User Interfaces - input devices and strategies, interaction styles

\section{General Terms}

Experimentation, Human Factors

\section{Keywords}

Gesture-based interaction, interface design, older users.

\section{INTRODUCTION}

Demographic, structural and societal changes in industrialized countries lead to a dramatic change in the percentage of elderly among the population: In Germany, for example, more than one third of the total population will be aged 65 and above within the next 30 years [1]. This development goes hand in hand with the dissemination of technology, and in particular mobile devices, into more and more areas of our daily living, where they become an integral part of work, education, healthcare, communication and entertainment [2]. This trend, however, puts people to a disadvantage who are facing problems using this technology, as in the case of many older adults. Thus, while technology in principle could compensate some of the age-related changes in their

Copyright is held by the author/owner(s).

MobileHCI’09, September 15 - 18, 2009, Bonn, Germany.

ACM 978-1-60558-281-8. physical, social and cognitive resources, thereby enhancing their quality of life, the elderly in many cases get disinterested and frustrated by too complex technology. Besides strategies to motivate and train older users to use technology, there is also a need for devices that are better tailored to the capabilities of an aging user. The normal aging process is typically accompanied with visual and auditory impairments, and a decline in working memory, selective attention, and motor control is observed [3].

\section{GESTURE-BASED INTERACTION}

In the domain of mobile devices, two recurring problems concerning elderly users are insufficiently sized displays and too small buttons, often confusingly labeled with multiple functions. Recent mobile devices that rely only on a relatively large touchsensitive screen as the main interface can theoretically meet these problems as text and icons can be displayed reasonably large, and buttons are in principle scalable. The benefit of touch screen interfaces over indirect input devices for elderly users has already been demonstrated (e.g. [4]). However, this technology comes at the cost of losing haptic feedback, and the problem of finding the appropriate commands from hierarchical menus still remains. The use of gestures might be a way to further facilitate interaction, and increase accessibility for older adults, as long as they are designed to match patterns and symbols the elderly are familiar with, and their execution is tolerant to the motor constraints an older user could bring about. Mobile devices which could register simple and familiar finger gestures, such as ticking off an item to confirm, or crossing out an item to delete (see Figure 1), might be able to facilitate interaction for the elderly. In this context, a gesture is understood as a meaningful pattern of finger movement on the touch surface. To date, it remains unclear whether such kind of finger interaction can constitute a real benefit for older users. Apart from open questions concerning the acceptance and adoption of such a new input paradigm among elderly users, it has also not been tested yet to which extend the motor capabilities of older adults [5] match the requirements of gesture input, especially if the gesture patterns get more complex.

\section{STUDY}

\subsection{Study Design and Participants}

In order to shed more light onto this last issue, the present study was designed to address the question of how the available gesture space and moreover the complexity of the gestures itself influences the performance of gesture execution. We were particularly interested in whether and how age moderates these dependencies. We compared 18 younger (21-33 years, mean 26 
years; SD: 3.4 years, $9 \mathrm{~m} \mathrm{9w}$ ) and 18 older adults (60-71 years, mean 64 years; SD: 3.2 years, $9 \mathrm{~m} 9 \mathrm{w}$ ) on a task of gesture imitation, measuring the speed and accuracy of gesture execution. After a short visual presentation of the gesture pattern, the participants' task was to retrace the gesture on a 15 " touchscreen monitor as fast and accurate as possible. The available space in which to draw the gesture was varied in three different sizes (small, medium, large), and gesture complexity was varied according to the number of lines that made up a gesture (single, double, multi-lined). In total, the gesture set comprised 42 different gesture patterns which were designed to match existing and suggested gestures for current and future mobile devices. The gestures spanned from simple linear movements, over circular gestures, to more complex, compound patterns.
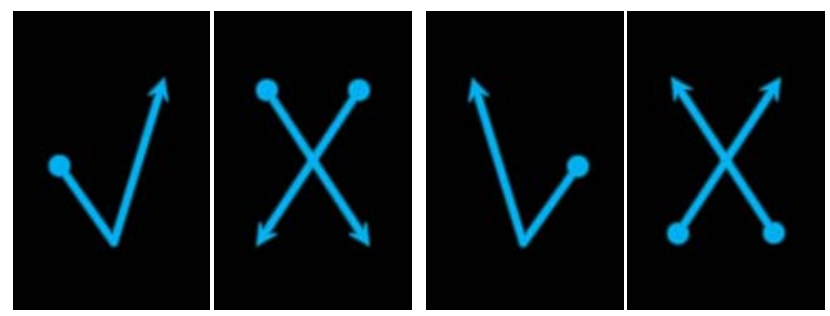

Figure 1: Example gestures. Familiar (left) and unfamiliar (right) versions

\subsection{Results}

Results showed that older users were about 1.3 times slower in their general performance than younger users $[F(1,34)=6.670$, $\mathrm{p}=.014]$. More important than speed, however, with respect to user interface design, is the accuracy of gesture execution. On measures of mean deviation to the best fitted line (or best geometrically fitted circle), as well as on angular deviation from reference angles, no significant influence of age could be observed. Even though systematic size influences on these measures could be observed (with larger gestures, also deviations got naturally larger), no interaction with age could be found. This indicates, that accuracy on this gesture set over all is achieved over different sizes and complexities - to similar levels by both younger and older users. Nevertheless, there were some interesting observations on the level of singular gestures: while most gestures consisted of simple geometric patterns (lines, edges, triangles, circles etc.), some also had semantic extensions, like the ticking off and crossing out gesture (see Figure 1), which were present in a normal, and a mirrored version. Significant differences were found between the familiar and the unfamiliar execution regarding accuracy, measured by angular deviation $[\mathrm{F}(1,34)=18.53, \mathrm{p}<.01]$. Interestingly, there was a significant interaction with age $[\mathrm{F}(1,34)=4.78, \mathrm{p}=.036]$, with older participants showing a much larger mean difference in angular deviation $\left(2.5^{\circ}\right)$ between the more familiar and the unfamiliar pattern than younger participants $\left(1.1^{\circ}\right)$. A similar effect could be shown in the execution speed, where the familiar versions were generally executed faster $[\mathrm{F}(1,34)=5.97, \mathrm{p}=.02]$. No age effects on accuracy were observed when only the direction of the gesture (up vs. down, or left. vs. right) varied between otherwise identical gestures.

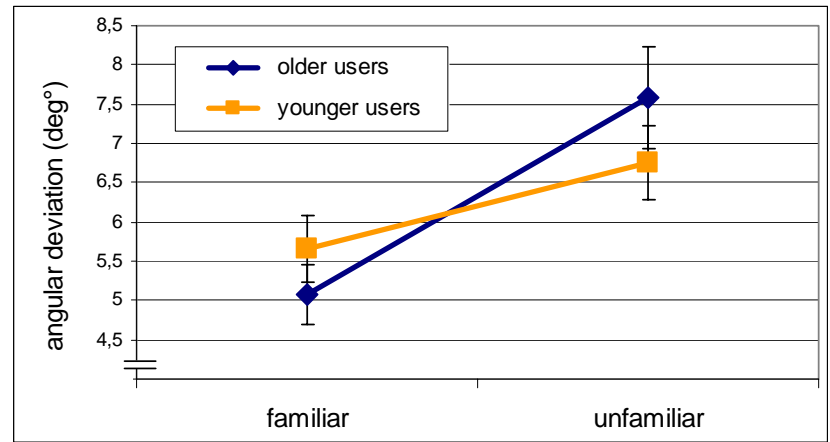

Figure 2: Accuracy of gesture performance (angular deviation in $\operatorname{deg}^{\circ}$ ) for familiar and unfamiliar gestures

\section{CONCLUSIONS AND OUTLOOK}

The results of this study indicate that older users (at least in the described age range) might be slower, but not necessarily less accurate performing simple gestures on a touch screen. Even small screen sizes (corresponding to the size of a PDA or mobile phone screen) are not particularly problematic. However, other factors, such as for example the familiarity of certain gesture patterns, can influence the performance, and elderly users seem to benefit especially from familiar patterns. This assumption should be validated in a follow-up study in which familiarity of gestures is experimentally controlled. Furthermore, a study testing a similar gesture set on a mobile device (iPod Touch) is currently carried out, which in addition also examines multi-touch gestures. With the second study, we investigate the generalization of these results to mobile devices. Besides, a special focus will be put on how the posture of the device influences gestures execution, since younger users are likely to operate a handheld device differently (e.g. making much more use of their thumps) than older users. Taken together, these results are a first step in the evaluation of gesture-based interfaces for elderly users, and might contribute to the design of interfaces which will suit the needs and abilities of older adults.

\section{ACKNOWLEDGMENTS}

This research is supported by the German Research Foundation, (DFG - 1013 'Prospective Design of Human-Technology Interaction') and by the National Research Fund Luxembourg, FNR (AFR grant).

\section{REFERENCES}

[1] Statistisches Bundesamt. 2006. 11. Koordinierte Bevölkerungsvorausberechnung Variante 1-W1.

[2] Czaja, S.J. and Lee, C.C. 2007. The impact of aging on access to technology. Universal Access in the Information Society. 5, 4 (April 2007), 341-349.

[3] Fisk, A.D., Rogers, W.A., Charness, N., Czaja, S.J., and Sharit, J. 2004. Designing for older adults. CRC Press, Boca Raton, USA.

[4] Murata, A. and Iwase, H. 2005. Usability of touch-panel interfaces for older adults. Hum Factors. 47, 4, 767-776.

[5] Vercruyssen, M. 1997. Movement control and speed of behaviour, in Human Factors and the Older Adult, A.D. Fisk and W.A. Rogers, Editors. Academic Press, San Diego. 55-86. 